\title{
Seeking an Understanding with Silicones
}

\author{
James P. Lewicki
}

Received: 26 June 2014 / Accepted: 6 October 2014 / Published online: 18 December 2014

(c) Springer Science+Business Media Dordrecht 2014

\section{RECEIVED DATE}

In 1973 my Father was at university studying microbiology. In one of his chemistry courses, his lecturer had generously devoted a day to synthetic polymers. Within that small segment, the topic of polysiloxanes came up very briefly and was summarized in a line; "Silicones show great potential". That one-liner stuck in my Father's head and years later he would still use it as an example of how far we had come technologically in a world now filled with all manner of advanced synthetic polymers - including the ubiquitous, yet forever misnamed 'Silicones'.

The anonymous lecturer from Heriot-Watt who made this statement in the 1970's was right. Silicones did show great potential, still $d o$ today and the tale of how they got everywhere from shampoo to Space is an interesting one that's much better off told by more authoritative sources than myself. In all honesty, I'm still not sure what polysiloxanes and their various derivatives even are from a physical standpoint at times, despite working with them my whole career. My lack of understanding of all aspects of their true nature is one of these nagging little things that surfaces now and again but I never quite capture. And it probably says more about my misspent university days and continuously increasing paperwork load than anything else. Nevertheless, I have decided to try and formalize my thoughts on at least one aspect of their properties in this brief editorial for our Special Issue on Silicones and Silicone Modified

J. P. Lewicki $(\bowtie)$

Lawrence Livermore National Laboratory, 7000 East Ave., Livermore, CA 94550, USA

e-mail: lewicki1@1lnl.gov
Materials and discuss one of the interesting aspects of polysiloxane elastomers - their uniquely poor tear strength.

PDMS - Why so fluid? Linear polysiloxanes such as polydimethylsiloxane (PDMS) show remarkable and often highly ideal behavior from a polymer physics standpoint. PDMS has one of the lowest glass transition temperatures of any synthetic polymer at $\sim-123^{\circ} \mathrm{C}$. The large Si-O-Si bond angle $\left(\sim 145-160^{\circ}\right)$, the low energy of rotation around the $\mathrm{Si}-\mathrm{O}$ bond and the relatively long $\mathrm{Si}-\mathrm{O}$ and $\mathrm{Si}-\mathrm{CH}_{3}$ bond lengths in PDMS mean that the polymer chain is extremely flexible and mobile. PDMS has a large molar volume, low energy polymer-polymer interactions and the polymer chains can coil and uncoil very freely when compared with something like an amorphous polyolefin. Of course, it is this very flexibility and freedom of motion that also allows PDMS to crystalize rapidly to as much as $98 \% \mathrm{w} / \mathrm{w}$ at only $-55^{\circ} \mathrm{C}$. And to even see amorphous PDMS below $T_{g}$ one must quench the polymer at rates in excess of $100^{\circ} \mathrm{C} / \mathrm{min}$. But at room temperatures, PDMS is most certainly a liquid - broadly irrespective of its molar mass. For a polymer, that's quite an achievement and it's often overlooked. In Fig. 1, linear PDMS having an average molar mass of $\sim 500$ Kda still behaves as a Newtonian fluid, albeit with a large time dependency: High molar mass PDMS will behave as a pseudo-solid over short timescales - even to the extent of retaining a fingerprint impression for several hours before the fluid surface evens itself out again. But leave a cube of it on the bench over the weekend and all you will have to show for it on Monday is a dark oily stain. From a viscoelastic perspective, the elastic response of even a highly entangled PDMS high polymer is minimal compared with 


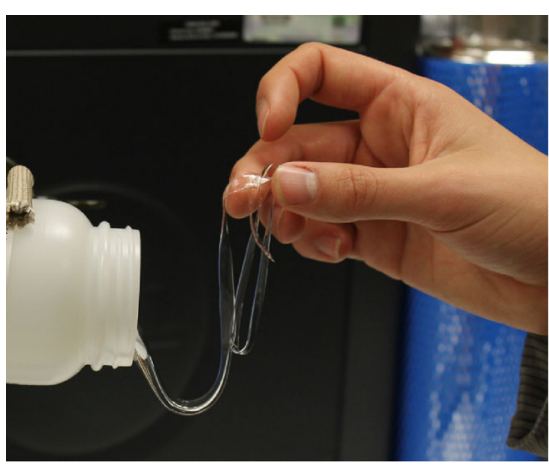

Fig. 1 Slow but still flowing. Unlike polyolefins, high molecular weight silicones, despite flowing slow enough to be picked up mid drip - are still Newtonian fluids at room temperature

its viscous dynamic component and this is clearly reflected in its interesting rheological behaviour.

Silicone rubber - a chemically pinned liquid? High molar mass siloxanes can be used to make all sorts of really interesting and powerful demonstrations of simple polymer dynamics. But from a practical standpoint, things get a lot more interesting when you crosslink polysiloxanes to form networks. Such 'silicone rubbers' are possibly one of the most commonly encountered and useful forms of polysiloxanes in our world today, but they have a few very interesting foibles that aren't always considered at first glance. Like all network elastomers, polysiloxane rubbers consist of polymer chains above their $T_{g}$ chemically or physically crosslinked to prevent viscous slippage leading to creep or even flow. Crosslink a siloxane into a simple 3D network and you get a compliant, flexible, optically transparent, gas permeable material that is practically useless in terms of its mechanical strength. You can tear, abrade and otherwise mechanically abuse such networks with little effort and the only mainstream way to render such materials mechanically robust is to 'fill' them to high loadings with a high modulus, heterogeneous secondary phase - such as fumed silica micro-particles. There is hardly a commercial silicone rubber product on the market today that is not filled in some way and compounded into a composite for application. And indeed, the association between polysiloxane networks and silica is so universal - that researchers have even been known to forget that the silica is even there and assume that the properties they observe are in fact native to the polysiloxane network alone! Indeed such 'common sense' thinking and the black-box like nature of commercial silicone elastomer formulations present a very real challenge in my own field of polymer aging and materials lifetimes. For example, unpicking the structure of a commercial formulation no longer manufactured, is an essential but non-trivial part of lifetime prediction and replacement material design efforts and it pays to be mindful of the initial intent of the formulation chemist when considering the potential structures of such materials.

What's less well discussed however is just why the tear strength of a simple polysiloxane network elastomer is so poor. It's not something I've ever heard or really formulated a succinct answer to, but let's give it a shot. Essentially - when you crosslink a polysiloxane via an end-linking process with a multifunctional molecule, you're joining together and binding often large, highly flexible chains that are significantly into the melt phase and have as we discussed earlier, no strong intra-molecular interactions beyond entanglement moderated by a high degree of chain flexibility. I like to think of end-linked polysiloxane networks as liquids that have been pinned down in a few places by the occasional chemical bond. Mechanically such a network has no chance of withstanding any significant shearing forces and will yield and fail according to its weakest macroscopic defects. You could go into a lot more depth, but essentially it seems to me that it's a polysiloxane's own chain flexibility that is its mechanical undoing.

A final thought. When you rip a PDMS model network in half to prove to yourself that it is indeed easy when there's no silica in there - you're bound to have broken a few chemical bonds in the process. As a polymer degradation chemist - I'd be interested to know what bonds broke and what formed in their place. It may yield further insight into the failure mechanisms involved.

There are a many more interesting macroscopic properties (and oddities) to polysiloxanes but it's safe to say that they all arise from the underlying structure and chain dynamics of the polymer systems. I think it's a fascinating subject both academically, practically and I'd like to think that researchers and technologists will continue to consider and study polysiloxanes from a polymer physics standpoint over the coming decades. We hope you enjoy this special issue on Silicones and Silicone Modified Materials and I hope that it highlights some of the novelty and new momentum in this diverse area of polymer science. 